\title{
Theoretical efforts on the understanding of solid- state systems based on polyoxometales grafted on carbon substrates for energy storage applications
}

\begin{abstract}
Abbreviations: POM, polyoxometalates; GO, graphene oxides; MWCNT, multi-walled carbon nanotubes; RGO, reduced-gos; SWCNT, single walled carbon nanotube; DFT, density functional theory
\end{abstract}

\section{Introduction}

A group of cluster metal oxides known as polyoxometalates (POM), present a large variety of configurations with the chemical formula $[\mathrm{MOx}] \mathrm{n}$, where $\mathrm{M}$ represents $\mathrm{Mo}, \mathrm{W}, \mathrm{V}, \mathrm{Nb}$ atoms and $\mathrm{X}$ ranges from 4 to $7 .{ }^{1}$ The charged versions of the POMs may be found with two different atoms; i.e., the heteroatom and the addenda atom. This subdivides the classification into two types: The Keggin and Dawson POMs. The former follow the notation $\left[\mathrm{XM}_{12} \mathrm{O}_{40}\right]^{\mathrm{n}}$-, whose nomenclature may be simplified to XM12. The latter, present the form $\left[\mathrm{X}_{2} \mathrm{M}_{18} \mathrm{O}_{62}\right]^{\mathrm{n}}$-, with $\mathrm{X}$ representing the heteroatom and $\mathrm{M}$, the addenda atom. Particularly, POMs of Keggin-symmetry are recognized to be used in devices of energy storage such as super capacitors and Liion batteries, catalysis and renewable energy applications. ${ }^{2}$ POMs present in such systems are susceptible to transfer electronic charge and to anchor at organic or inorganic substrates. This opens a field of research to design novel materials to be implemented on electrodes of solid state. In this respect, the tailoring of POMs adsorbed on carbon substrates aids in the development of improved devices. A technique to achieve this goal is the chemisorption of POMs on carbon substrates. ${ }^{3,4}$ This technique is developed through the oxidation of the carbon substrate, and it may be extended to multi-walled carbon nanotubes (MWCNT), ${ }^{5}$ activated carbon, ${ }^{6}$ carbon nanofibers ${ }^{7}$ or graphene. For instance, Zhou and $\mathrm{Han}^{8}$ found a route to obtain a composite material with a strong interaction between a $\mathrm{PMo}_{12}$ polyoxometalate and a graphene sheet. This material reported a larger surface area than that found on graphene oxides (GO) and reduced-GOs (RGO). The increase in electron redistribution reported on this system was used to achieve controllable Hydrogen adsorption-desorption properties. On the other hand, the synthesis of $\mathrm{PMo}_{12}, \mathrm{PW} 12$ and PMo11V supported on RGO and alkyl-functionalized RGO sheets have also been reported. ${ }^{9}$ It was evidenced that this interaction changes the electronic properties of POMs, such as a linear-shift reported on the metal-oxygen vibrational frequency, which depends on the loading of the POMs. Besides, as a consequence of the POMs dispersion on the graphene sheets, they resulted less oxidized, indicating that the POMs are sensitive to the environment, and particularly to the interaction with the support. Kume et al. ${ }^{10}$ reported the synthesis of $\mathrm{PMo}_{12}$ adsorbed on an RGO surface. This ensemble showed a better performance as a cathode in a molecular cluster battery, than that of a POM adsorbed on a single walled carbon nanotube (SWCNT) or with a POM isolated system. This addresses a peculiar increasing on the capabilities of the RGO as a capacitor, which is directly induced by the presence of the adsorbed POM. Nevertheless, such enhancement could not be explained from experimental grounds. Additionally, Li et al. ${ }^{11}$ synthesized RGO by using an UV-irradiated photo-reduction process, where $\mathrm{PMo}_{12}$ acted as a photocatalyst. This POM was also adsorbed on the RGO surface and yielded the graphene layers as water-dispersible.

\author{
Volume I Issue 3 - 2017
}

\author{
Jesús Muñiz ${ }^{1,2}$ \\ 'Instituto de Energías Renovables, Universidad Nacional \\ Autónoma de México \\ ${ }^{2}$ CONACYT-Universidad Nacional Autónoma de México
}

Correspondence: Jesus Muniz, Instituto de Energias Renovables, National Autonomous University of Mexico, Priv. Xochicalco s/n, Col. Centro, Temixco, Morelos, Mexico, Tel +52 777362 0090,Email jms@ier.unam.mx

Received: May 31, 2017 | Published: August 03, 2017

Nevertheless, the mechanism behind the bonding of POMs on carbon substrates has been obscure, and it has been not clearly understood if the presence of functional groups is a necessary condition to assure POM grafting. Consequently, the study of POMs at carbon interfaces from a theoretical point of view appears to be a solution to shed light into the understanding of the mechanisms behind bonding and electronic structure properties involved with energy storage. In this regard, Wen et al. ${ }^{12}$ studied the system PW12 adsorbed on graphene using Density Functional Theory (DFT), which refers to the resolution of the Many-Body Schrödinger equation, which is intended to find the electronic density of a system to obtain all physico-chemical parameters observed in a molecular system. In the cited work, the charge transfer between the POM and carbon was revised, and a charge transfer from the POM to graphene, was anticipated. The system $\mathrm{PMo}_{12}$ adsorbed on a CNT with the presence of TBA $=\left(\mathrm{C}_{4} \mathrm{H}_{9}\right)_{4} \mathrm{~N}+$ was also studied. ${ }^{13}$ In this case, the inverse mechanism of charge transfer was found. On the other hand, our group ${ }^{14}$ theoretically studied at DFT level, the $\mathrm{PMo}_{12}$ cluster adsorbed on graphene and on $\phi \mathrm{X} /$ graphene, where $\mathrm{X}$ corresponds to the functional groups $-\mathrm{OH}$ and $-\mathrm{NH}_{2}$. In this work, a non-covalent attraction of the electrostatic-type was evidenced at the interface POM/ graphene. Those systems where the $\mathrm{PMo}_{12}$ cluster was anchored to the functionalized $\varphi$-X/graphene with higher energies resulted to be the composite systems where the electronic charge is more susceptible to remain fixed. This may provide an electrode made of such materials, the capacity to sustain strengthened density currents in charge/ discharge cycles observed in energy storage devices. An analogous work was also performed ${ }^{15}$ on the series $\left[\mathrm{XM}_{12} \mathrm{O}_{40}\right]^{\mathrm{n}}$ - adsorbed on graphene. In this case, $\mathrm{X}=\mathrm{Pd}, \mathrm{P}, \mathrm{Ru}, \mathrm{Si}$ and $\mathrm{M}=\mathrm{Mo}, \mathrm{Nb}$ and $\mathrm{W}$. The magnitude of its charge may correspond to 2,3,4 and 8. A benchmark analysis was performed to test different DFT methodologies. Besides, the non-existent clusters $\mathrm{PdMo}_{12}$ and $\mathrm{RuNb}_{12}$ were predicted, since our computational tools allow us to find novel chemical structures that may be stable upon chemical synthesis. The results showed that the incorporation of POMs onto graphene may open new pathways that the electrons may track in the charge/discharge processes mentioned above. On the other hand, such DFT studies have also been carried 
out on the $\left[\mathrm{PW}_{11} \mathrm{O}_{39}\right]^{7}$ - cluster adsorbed on $\mathrm{Au}(100)$ and $\mathrm{Ag}(100)$ surfaces. ${ }^{16}$ This gives evidence on the adsorption of POM clusters onto pure metallic surfaces. From another theoretical perspective, namely Molecular Dynamics, the adsorption of POMs on surfaces has been studied. This methodology is aimed to solve Newtonian Mechanics equations of a certain atomistic system, with the advantage to include a larger number of atoms in the calculation. At this level of theory, ${ }^{17}$ the $\mathrm{SiW}_{12}$ cluster adsorbed on a silver substrate was analyzed. In this case, the reduction of the POM was calculated in the presence of water solvent molecules. Finally, Rozanska et al. ${ }^{18}$ simulated using DFT, the $\mathrm{PMo}_{12}$ cluster adsorbed on cristobalite and a bonding of covalent nature was found.

\section{Conclusion}

There are many open questions that still deserve full attention on the chemistry of POMs adsorbed on carbon substrates, such as the search of novel functional groups that would allow effective POM retention and the influence of solvent effects that may arise in aqueous electrolytes, in which super capacitors or batteries operate. This knowledge may bring new opportunities for multi-scale calculations, which may explain more complex phenomena present at experimental and industrial applications of energy storage.

\section{Acknowledgements}

None.

\section{Conflict of interest}

The author declares no conflict of interest.

\section{References}

1. Long DL, Tsunashima R, Cronin L. Polyoxometalates: Building Blocks for Functional Nanoscale Systems. Angew Chem Int Ed Engl. 2010;49(10):1736-1758.

2. Yuanchun JI, Lujiang H, Jun H, et al. Polyoxometalate-functionalized nanocarbon materials for energy conversion, energy storage and sensor Systems. Energy Environ Sci. 2015;8:776-789.

3. Kang Z, Wang Y, Wang E, et al. Polyoxometalates nanoparticles: synthesis, characterization and carbon nanotube modification. Solid State Commun. 2004;129:559-564.

4. Kulesza PJ, Skunik M, Baranowska B, et al. Electrochemical properties of polyoxometalates as electrocatalysts. Chem Rev. 1998;98(1):219-237.
5. Fei B, Lu H, Hu Z, et al. Solubilization, purification and functionalization of carbon nanotubes using polyoxometalate. Nanotechnology. 2006;17(16):1589-1593.

6. Gallegos AKC, Cortina SL, Brousse T, et al. Electrochemical study of $\mathrm{H}_{3} \mathrm{PMo}_{12}$ retention on vulcan carbon grafted with $\mathrm{NH}_{2}$ and oh groups. Journal of Solid State Electrochemistry. 2016;20(1):67-79.

7. Guevara JS, Ruiz V, Romero PG. Hybrid Energy Storage: High Voltage Aqueous Supercapacitors Based on Activated Carbon-Phosphotungstate Hybrid Materials. J Mater Chem A. 2014;2(4):1014-1021.

8. Zhou D, Han BH. Graphene-Based Nanoporous Materials Assembled by Mediation of Polyoxometalate Nanoparticles. Advanced Functional Materials. 2010;20(16):2717-2722.

9. Tessonnier JP, Goubert RS, Alia S, et al. Structure, stability, and electronic interactions of polyoxometalates on functionalized graphene sheets. Langmuir. 2013;29(1):393-402.

10. Kume K, Kawasaki N, Wang H, et al. Enhanced Capacitor Effects in Polyoxometalate/Graphene Nanohybrid Materials: A Synergetic Approach to High Performance Energy Storage. J Mater Chem A. 2014;2:3801-3807.

11. Li H, Pang S, Feng X, et al. Polyoxometalate assisted photoreduction of graphene oxide and its nanocomposite formation. Chem Commun (Camb). 2010;46(34):6243-6245.

12. Wen S, Guan W, Wang J, et al. Theoretical investigation of structural and electronic properties of [pw12o40]3- on graphene layer. Dalton Trans. 2012;41(15):4602-4607.

13. Wen S, Guan W, Kan Y, et al. Theoretical insights into [PMo12O40]3grafted on single-walled carbon nanotubes. Phys Chem Chem Phys. 2013;15(23):9177-9185.

14. Muniz J, Gallegos AKC, Robles M, et al. Bond formation, electronic structure, and energy storage properties on polyoxometalate-carbon nanocomposites. Theor Chem Acc. 2016;135:92-109.

15. Muniz J, Celaya C, Ozuna AM, et al. First principles study on the electronic structure properties of Keggin polyoxometalates on carbon substrates for solid-state devices. Theor Chem Acc. 2017;136:26.

16. Lang Z, Aparicio XA, Weinstock I, et al. Counterintuitive adsorption of [PW11O39]7-on Au(100). Inorg Chem. 2017;56(7):3961-3969.

17. Aparicio XA, Miro P, Clotet A, et al. Polyoxometalates adsorbed on metallic surfaces: immediate reduction of [SiW12O40]4-on $\mathrm{Ag}(100)$. Chem Sci. 2012;3(6):2020-2027.

18. Rozanska X, Sautet P, Delbecq F, et al. Polyoxometalate grafting onto silica: stability diagrams of H3PMo12O40 on $\{001\},\{101\}$, and $\{111\}$ $\beta$-cristobalite surfaces analyzed by DFT. Phys Chem Chem Phys. 2011;13(35):15955-15959. 